\title{
Lidil
}

Revue de linguistique et de didactique des langues

$50 \mid 2014$

Variation stylistique et diversité des contextes de socialisation

\section{Christel Troncy (dir.) et Jean-François De Pietro, Livia Goletto et Martine Kervran (coll.), Didactique du plurilinguisme: approches plurielles des langues et des cultures. Autour de Michel Candelier}

Presses universitaires de Rennes, 2013

Chantal Dompmartin-Normand

\section{OpenEdition}

Édition électronique

URL : http://journals.openedition.org/lidil/3654

DOI : $10.4000 /$ lidil.3654

ISSN : 1960-6052

Éditeur

UGA Éditions/Université Grenoble Alpes

Édition imprimée

Date de publication : 15 décembre 2014

Pagination : 213-215

ISBN : 978-2-84310-287-5

ISSN : $1146-6480$

Référence électronique

Chantal Dompmartin-Normand, « Christel Troncy (dir.) et Jean-François De Pietro, Livia Goletto et Martine Kervran (coll.), Didactique du plurilinguisme : approches plurielles des langues et des cultures. Autour de Michel Candelier », Lidil [En ligne], 50 | 2014, mis en ligne le 15 juin 2016, consulté le 25 septembre 2020. URL : http://journals.openedition.org/lidil/3654 ; DOI : https://doi.org/10.4000/lidil. 3654 


\section{Notes de lecture}

Christel Troncy (dir.) et Jean-François De Pietro, Livia Goletto et Martine Kervran (coll.), Didactique du plurilinguisme : approches plurielles des langues et des cultures. Autour de Michel Candelier, Presses universitaires de Rennes, 2013

Ce volumineux ouvrage (513 pages) rassemble les contributions de nombreux auteurs autour de Michel Candelier (dorénavant MC) et de son travail de recherche dans le champ de la didactique du plurilinguisme et des approches plurielles. C'est un ouvrage de facture particulière puisqu'il se propose d'être un volume d'hommages, tout en mettant en discussion et en dialogue des écrits sélectionnés de MC et les textes des autres contributeurs.

L'ouverture propose des réflexions didactiques «autour des approches plurielles », rappelant qu'avant leur naissance, le chemin de MC a été marqué par la rencontre avec l'équipe de Louise Dabène à Grenoble. À partir de là, le travail amorcé sur l'éveil aux langues dans les années 1980, sur les traces de l'Awareness of language en provenance d'Angleterre, va constituer un matériau de pavage essentiel des «chemins en didactique ${ }^{1} »$ que construira et empruntera MC tout au long de sa carrière prolifique. Les fondations de ses chemins sont d'abord «l'engagement contre l'isolationnisme monolinguistique» (p. 25) et le souhait de promouvoir une «éducation linguistique globale polycentrique s'appuyant sur de nombreuses langues reliées les unes aux autres par un échange soutenu» (p. 25). Ce qu'il construira sur ces fondations constitue une contribution majeure à la création du nouveau champ des approches didactiques plurielles des langues et des cultures (dorénavant AP).

En ouverture de l'ouvrage, C. Troncy resitue pour le lecteur les dynamiques terminologiques et définitoires traversant le champ. Retenons que les approches plurielles prônent «le travail conjoint sur plus d'une

1. Son HDR en 1994 est intitulée «Chemins en didactique. Pour le plurilinguisme à l'école». 
langue» avec une réflexion métalinguistique, que la didactique du plurilinguisme s'inscrit dans «l'éducation plurilingue et interculturelle» selon les termes du Conseil de l'Europe, que le point focal est le développement de la «compétence plurilingue et pluriculturelle» des apprenants, tout cela constituant un renversement de paradigme par rapport à la conception de la didactique des langues, séparées les unes des autres dans des «approches singulières».

Les chapitres proposent à chaque fois des articles réédités de MC et co-auteurs, qui sont mis en regard de contributions d'autres auteurs, éclairant différents contextes et réflexions. Des articles illustrent en première partie des démarches concrètes où le lecteur peut comprendre mieux ce que recouvrent les AP et à quoi elles servent. Des démarches d'éveil aux langues par exemple sont mises en œuvre avec profit pour développer un rapport affectif, esthétique et sensible aux langues et contribuer à la construction identitaire (J.-F. Bourdet, J. Aden, F. Leclaire), pour «déconstruire l'insécurité linguistique dans les espaces sociaux de pluralité linguistique inégalitaire»(A. Bretegnier), pour activer «des dynamiques relationnelles» entre adolescents de lycée professionnel (P. Lambert), pour contribuer à la construction de sociétés plurielles et solidaires (F. Armand).

La question de l'insertion de l'éveil aux langues dans les curriculums est abordée ainsi que celle de l'articulation entre les approches : articulations entre AP et enseignement bilingue (M. Cavalli), articulation «raisonnée et pragmatique» pour une éducation «de qualité » (F. Heyworth), «convergences» à chercher (J. Billiez et D.-L. Simon) en prenant finement en compte les contextes où ces approches s'insèrent. Les AP ne s'opposent pas aux approches singulières, mais doivent s'y articuler : «[...] les AP découlent d'abord d'un état d'esprit : aborder quelqu'objet qui soit dans une perspective ouverte - plurielle - quitte à "resserrer" vers le singulier lorsque cela est nécessaire ou plus opératoire [...].» (J.-F. De Pietro, p. 236)

Toutefois la diffusion des AP dans les systèmes éducatifs constitue encore un défi dans bien des cas, malgré la richesse des outils et démarches utilisables en formation d'enseignants en particulier, ce dont nous parle la deuxième partie. C'est un défi stimulant, si elles sont une «utopie sociale» (chapitre 4), avec le terme «utopie» qui renverrait à la fécondité des actions, si l'on en croit C. Perregaux (p. 331). En effet, ce sont bien aussi des «effets sociaux» sous forme de cohésion sociale, qui sont à attendre des AP.

En clôture de volume, D. Coste joue sur le(s) pluriel(s) pour replacer la thématique dans l'histoire de la didactique des langues et des didac- 
tiques de langues, mettant par là subtilement à l'épreuve le chapeau «didactique du plurilinguisme» et proposant finalement «une approche plurielle des didactiques singulières », à savoir que "chaque didactique de langue prise isolément $[\ldots]$ est à même tout à la fois de développer $[\ldots]$ l'apprentissage $[\ldots]$ singulier de cette langue et d'offrir aux apprenants des occasions de contacts pratiques et réflexifs avec une ou plusieurs autres langues [...]. L'AP de la didactique singulière résulte de cette complémentarité entre le travail majeur de la langue visée et l'exposition construite à des formes langagières et culturelles autres».

Ce dernier exposé arrive à point nommé pour aider à la «digestion» de l'ensemble de l'ouvrage. Celui-ci est dense et documenté. C'est un bon outil pour le chercheur du domaine, car il rassemble nombre d'articles de référence et d'autres qui vont le devenir. Il convoque sans nul doute la perspicacité du lecteur, tant la thématique, passionnante au demeurant, boucle et reboucle sur des questionnements complexes, lesquels témoignent de la curiosité et de l'exigence de Michel Candelier et de ses compagnons sur les chemins de la didactique du plurilinguisme.

Chantal Dompmartin-Normand

Université Toulouse-Jean Jaurès, Université Grenoble Alpes, LIDILEM

Florimond Rakotonoelina (dir.), Perméabilité des frontières entre l'ordinaire et le spécialisé dans les genres et les discours, Les Carnets du Cediscor, n 12, Paris, Presses Sorbonne Nouvelle, 2014, 186 p.

S'appuyant sur deux décennies de travaux du Centre de recherche sur les discours ordinaires et spécialisés (CEDISCOR) autour des discours et des genres qualifiés comme «ordinaire» ou «spécialisé», ce numéro des Carnets $d u$ Cediscor approfondit ces deux notions, laissant une large place aux formes hybrides. Comme rappelé par F. Rakotonoelina dans l'avant-propos, les positions théoriques et méthodologiques qui traversent l'analyse de ces types de corpus ont connu un historique contrasté. L'analyse du discours spécialisé bénéficie d'une tradition forte. En contraste, le développement récent des technologies de la communication, ouvertes au grand public comme aux expert-e-s, a engendré des discours ordinaires, variés et hétérogènes. L'ouvrage présente six études rassemblées en trois parties et permettant de creuser les limites 